\title{
Simulator evaluation of the additional verification of intravenous drug (AVOID) -error system: A prospective randomized controlled study
}

\author{
Shazia Khan ${ }^{1}$, Pamela Chia ${ }^{2}$, Shariq Ali Khan ${ }^{3 *}$ \\ ${ }^{1}$ Senior Registrar, ${ }^{2}$ Senior Resident, ${ }^{3}$ Consultant, ${ }^{2,3}$ Dept. of Anaesthesia, ${ }^{1}$ Ophthalmology Service, KK Women's and Children's Hospital, \\ Singapore, ${ }^{2,3}$ Singapore General Hospital, Singapore \\ *Corresponding Author: Shariq Ali Khan \\ Email: shariq.ali.khan@singhealth.com.sg
}

Received: $19^{\text {th }}$ February, 2019

Accepted: $25^{\text {th }}$ April, 2019

\begin{abstract}
Introduction: Medication errors occur in around $0.75 \%$ to $5 \%$ of all anaesthesia related medication administrations. We developed the "Additional verification of intravenous drug (AVOID) -error System" to reduce medication-errors in Anaesthesia and tested the effect of this system on drug-administration time and end-user acceptance in a simulated environment.

Materials and Methods: A randomized-controlled cross-over trial study on 20 anaesthesiologists from Singapore General Hospital was conducted in a simulated operating-room environment in September 2018. Participants were randomized to administer five 1 millilitre doses, from a prefilled pre-labelled syringe with and without the AVOID-error System, (intervention group and control group, respectively). The primary outcome recorded was time to complete a "drug administration". Additionally, all study participants were individually asked to give an opinion about the effect of the AVOID-error system on workload, medication error reduction and willingness to use in their routine practice using a five-point Likert scale. A Paired t-test was used for inter-group comparison and statistical significance was defined as $\mathrm{p}<$ 0.05 .

Results: Twenty Anaesthesiologists, (12 consultants and 8 trainees) with median 8.5 years (IQR, 3.75-22) of experience participated in the study. The mean time to complete a drug administration in the intervention and control groups were, $18.2(\mathrm{SD} \pm 5.3)$ and $12.6(\mathrm{SD} \pm 3.8)$ seconds ( $\mathrm{p}=0.001)$, respectively.

Conclusions: On an average, the use of the Avoid-error system increased the total drug-administration time by 5.6 (95\% CI: 4.6 - 6.5 ) seconds.
\end{abstract}

Keywords: Human factors, Medical device, Medication error.

\section{Introduction}

The Anaesthesiologist is probably the only hospital physician that prescribes, prepares and administers medications to patients, with minimal or no secondary confirmation from another health professional. Additionally, the anaesthesiologist is also responsible for other cognitively demanding and distracting intraoperative tasks like observing patient vitals, dealing with emergency situations and performing a variety of procedures like transesophageal Echo. It is therefore not surprising that medication errors occur in around $0.75 \%$ to $5 \%$ of all anaesthesia related medication administrations. ${ }^{1-3}$ Considering the fact that more than 300 million inpatient surgeries are performed annually, ${ }^{4}$ more than 3 million patients are exposed to potential harm of medication errors every year, worldwide.

Substitution errors, like ampoule and syringe swaps, account for up to $60 \%$ of medication errors in anaesthesia ${ }^{1}$ and occur when an anaesthesiologist fails to follow the "Safe Drug Administration procedure" (defined as a two-step procedure where, step one is reading a drug-ampoule to select a correct label for a syringe and step two is reading the labelled syringe before administering it intravenously). Although commercial devices like the SLS 500i syringe Label System (Codonics, Middleburg Heights, OH, USA) and the SAFER sleep system (Safer Sleep, Auckland, New Zealand) have been developed to reduce the risk of substitution errors. Studies have found that, in a clinical environment, only $25-62 \%$ of anaesthetists follow the 'rule' of scanning the barcoded syringe label before drug administration. ${ }^{6,7}$ Thus both these systems continue to expose patients to the risk of syringe swap. ${ }^{8}$

Recently, we developed the "Additional verification of intravenous drug (AVOID) -error System" which includes a "lock-like" device that attaches to the patient's intravenous (IV) tubing, and allows injection of the drug only after the user performs a confirmatory scan of the barcode on the syringe label. Although the randomized controlled trial evaluating the first prototype found that the system was able to produce $100 \%$ compliance with the Safe Drug Administration procedure, ${ }^{5}$ Study participants and readers expressed concerns primarily about the drug administration time-delays and end-user acceptability. ${ }^{9}$ Taking these concerns into consideration we developed the next iteration of the AVOID-error system and tested it in the following randomized controlled study. The primary outcome measured was the difference in time taken for each drug administration using the AVOID-error system compared to control. Additionally, User feedback about impact on workload, patient safety and willingness to use were also ascertained using a structured questionnaire.

\section{Materials and Methods}

It was the opinion of the Institutional Research Ethics Board that Simulations Studies of this type do not require a formal ethical review. After obtaining written informed consent, 20 anaesthesiologists from Singapore General 
Hospital were invited to participate in this cross-over study conducted in a simulated operating room environment. Each study participant was instructed to administer five 1 millilitre doses, from a prefilled pre-labelled syringe with and without the AVOID-error System (Intervention and Control groups, respectively). A coin toss was used to randomly determine a participant's sequence of entry into the Intervention or Control groups. The primary outcome recorded was the time to complete a "drug administration". This was defined as the total time taken to complete the following sequence of actions: [1] picking up the syringe from the drug tray to injection of drug into the Intra-Venous (IV) port and then return of syringe to drug tray, [2] Documentation of drug details in the anaesthesia information management system (AIMS) (WinChart Health Informatics, Sydney, NSW, Australia). Special care was taken to maintain a standardized distance between the drug tray and injection port in both the study groups. Time taken to document drugs was measured from the point the participant first touched the AIMS user input console to the point the participant pressed 'enter' to complete the documentation. Additionally, all study participants were individually asked to answer questions about the effect of the AVOID-error system on workload, medication error reduction and willingness to use in their routine practice using a five point Likert scale (Table 1). This manuscript adheres to the applicable CONSORT guidelines.

\section{Statistical Analysis}

Based on a pilot study, we found that the average time taken for each drug administration event was 11.3 seconds (SD: \pm 3.9 ) during routine practice. It was our clinical opinion that an absolute difference of 5 seconds would be a clinically significant difference in drug administration times between intervention and control study groups. Therefore, to detect an absolute difference of 5 seconds in drug administration time (Primary outcome) between the intervention and control study groups with a power of $90 \%$ and an alpha error of 0.01 , 19 drug administration events would be required in each study group. All data were analysed using SPSS version 23.0 (IBM, Armonk, NY, USA). A Paired t-test was used for intergroup comparison and statistical significance was defined as $\mathrm{p}<0.05$.

Table 1: Participant feedback questionnaire about the AVOID-error system.

Q1: Compared to your routine practice of administering Intra-Venous drugs in the Operating Room, How do you think the AVOID-error system would impact your workflow?

O Significantly improve workflow [1]

O May improve workflow [2]

O No Impact on workflow [3]

O May Slow workflow [4]

O Significantly slow workflow [5]

Q2: By compelling labelling and confirmation scanning of syringes of drugs, do you think the AVOIDerror system can reduce the risk of medication errors?

O Significantly reduce errors [1]

O May reduce errors [2]

O No Impact on errors [3]

O May increase errors [4]

O Significantly increase errors [5]

Q3: Would you like to have the AVOID-error system your place of work, (if cost was not a consideration)?

O Definitely yes [1]

O Maybe yes [2]

O Neutral [3]

O Maybe no [4]

O Definitely no [5]

*For the benefit of the readers, numbers with '[]' brackets have been added to the questionnaire to indicate the score used for analysis. These numbers were not visible to the participants during the study. 


\section{Description of AVOID-Error System}

The Additional verification of intravenous drugs (AVOID) -error system, was developed with the hypothesis that a system which mandates the anaesthesia provider to verify intravenous drugs before administration would result in a reduction in the risk of medication error. The AVOIDerror system consists of the following components:

1. Touch screen Graphic user interface with a barcode scanner (Fig. 1a) mitigate potential infection control risks, this component further consists of a (a) disposable component and (b) reusable housing component.

3. Software, facilitating wireless communication between components $1 \& 2$ and with Anaesthesia information systems using application program interfaces.

The system has been designed to work either as a standalone system using prefilled-prelabelled syringes or with commercially available syringe label printers like the Codonics SLS 500i printer. ${ }^{5}$ Drug administration Workflow (Fig. 2) is designed to be user friendly, to encourage adoption. Some important features of the system are as follows:

\section{Emergency Drug Administration (EDA) Port (Fig. 1b)}

The EDA port allows the Anaesthesia provider to give intravenous medications to patients in case of system failure or in situations where a delay in drug administration by the AVOID-error system is deemed to be potentially dangerous to the patient. The AVOID-error system is able to detect drug administration through the EDA port and is able to notify the system administrators.
2. The AVOID-error patient intravenous port (Fig. 1b). To

\section{Ability to Prevent Accidental Administration of Allergic Medication}

The system locks "AVOID-error patient intravenous port" when the user scans a syringe label which contains an allergic drug. Thereby, preventing accidental administration. Ability to Prevent Accidental Sharing of Syringes between Patients

The system software uniquely "tags" the labelled syringes to a particular patient and prevents accidental use of that syringe in another patient.

Ability to Facilitate Timely Administration of Antibiotics with a Verifiable Audit Trail

Each drug administered using the AVOID-error system is time-stamped and entered in to the AIMS. This auditable data can be used to verify compliance with pre-incision antibiotic administration guidelines if needed.

\section{Future Development}

Some features that are being developed and maybe included in the next version of the system include:

1. Smaller form factor for the AVOID-error patient intravenous port: Although the device is currently relatively small, self-contained and around 30 grams. In the next iteration we plan to make it even lighter and smaller.

2. Administered drug volume sensing: In the next iteration that device will be able to sense the volume of fluid administered. Thus, making the process of documenting administered drug even more user friendly.

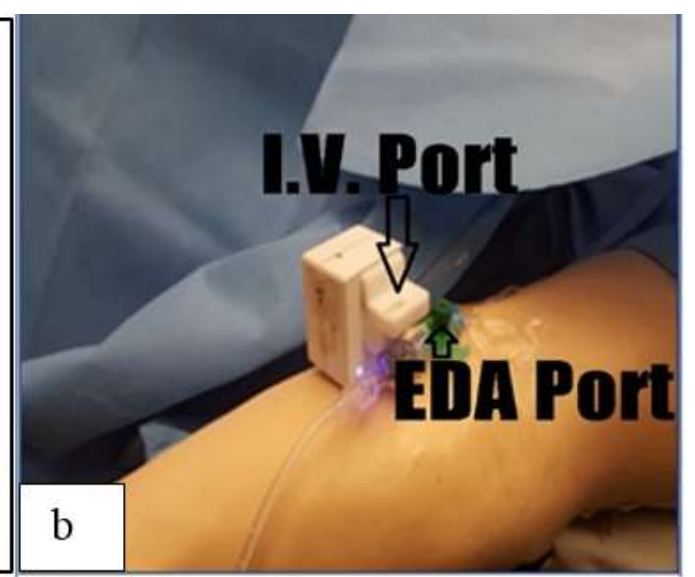

Fig. 1: Hardware components of the AVOID-error system. (1a): The AVOID-error system touch screen Graphic user interface with a barcode scanner. (1b) The AVOID-error patient intravenous port and emergency drug administration (EDA) port 


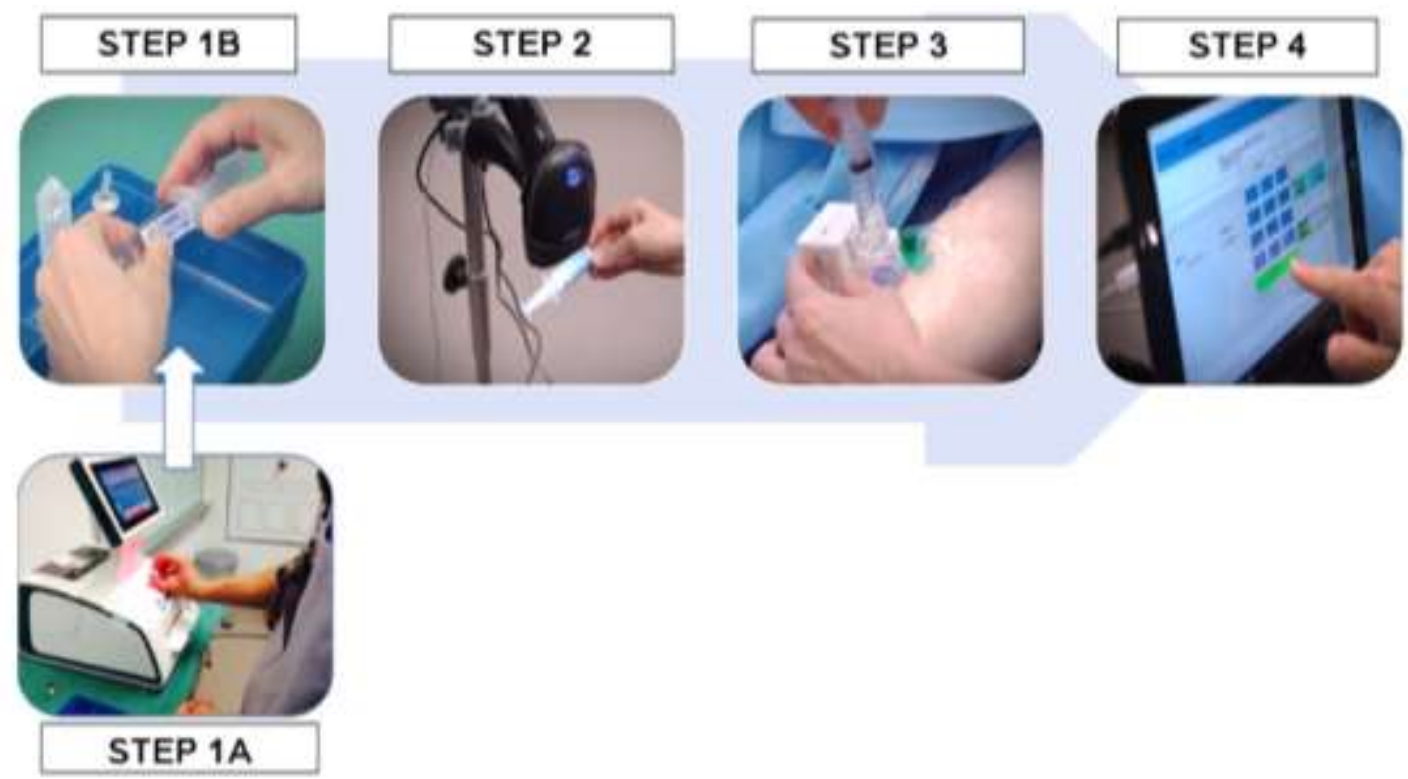

Fig. 2: The AVOID-error system drug administration workflow; Step 1A -1B: The end user scans the drug ampoule and prints the syringe label using a label printer OR uses Prefilled prelabelled syringes; Step 2: The system mandates scanning the syringe-label using the device scanner prior to drug administration. The scan produces an audio-visual cue consisting of drug name and concentration; Step 3: On successful completion of Step 2, the AVOID patient IV device unlocks and allows injection of syringe into the drug administration port; Step 4: After drug injection, the end-user enters administered drug amount into AVOID-error system

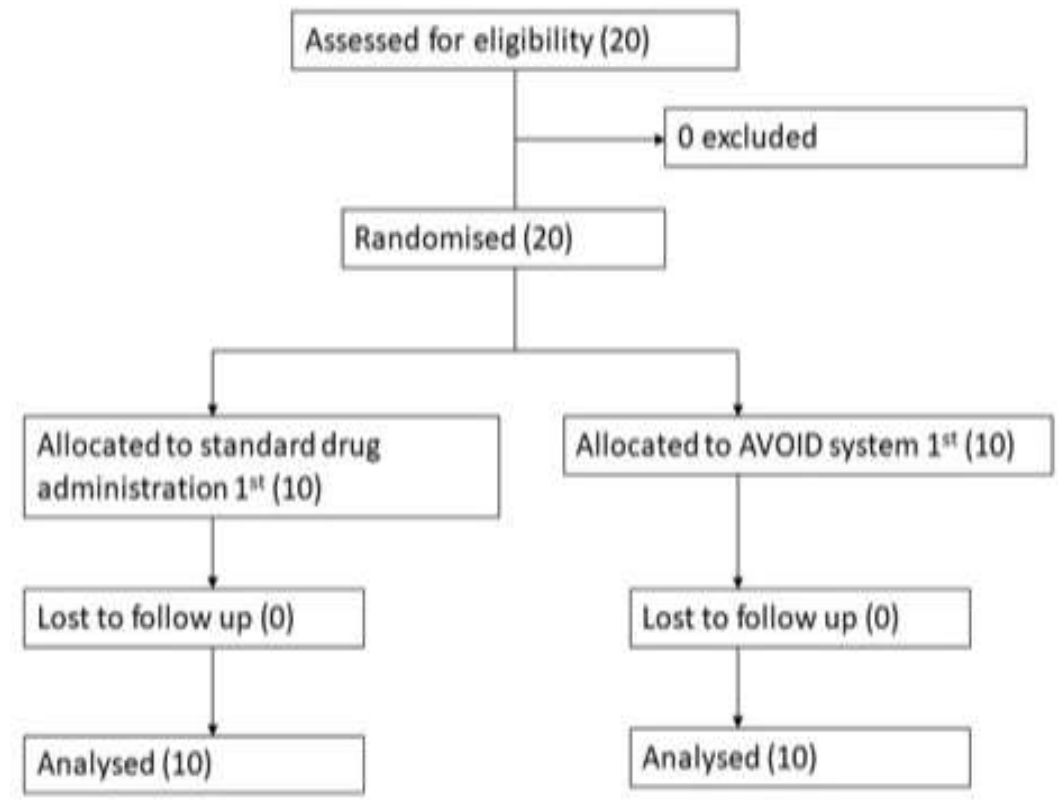

Fig. 3: Consort flow diagram for screening, inclusion, and exclusion of trial participants

\section{Results}

Twenty Anaesthesiologists, (12 consultants and 8 trainees) with median 8.5 years (IQR, 3.75 - 22) of experience participated in the study (Fig. 3). The study was conducted over a period of two days and each participant was asked to perform 5 drug administrations in the intervention and control study groups, there were a total of 200 drug administrations performed in the study (100 each in the intervention and control groups respectively).

The mean time to complete a drug administration in the intervention and control groups were, 18.2 (SD \pm 5.3 ) seconds and $12.6(\mathrm{SD} \pm 3.8)$ seconds $(\mathrm{p}=0.001)$, respectively. On an average, the use of the AVOID-error system increased the total drug administration time by 5.6 seconds $(95 \% \mathrm{CI}$, $4.6-6.5)$.

Participant feedback about the AVOID-error system was obtained at the end of the study (Fig. 4), on response to the effect of the AVOID-error system to workload, most 
participants were in the opinion that the system "May slow workflow" (median, grade 4 (IQR, 3-4)). All the participants agreed that the use of the AVOID-error system may lead to a reduction in medication errors (median, grade 2(IQR, 1- 2)). Finally, most participants were willing to use the AVOIDerror system in their routine practice (median, grade 2 (IQR, 2-3)).

\section{Discussion}

We found that the time to complete a drug administration using the AVOID-error system was 5.6 seconds (95\% CI, 4.6 - 6.5) slower compared to control.

Around two-third of medication errors in Anaesthesia can be attributed to substitution errors, like accidently drawing up a drug from an incorrect ampoule (= ampoule swap) or accidentally injecting a drug from the wrong syringe (= syringe swap). ${ }^{1,8}$ Clinical Studies have found that existing commercial devices, like the SAFERSleep System and the Codonics SLS 500i, are not very successful in promoting the anaesthesia provider to counter check syringe labels prior to drug administration. ${ }^{6,7}$ Thus, the patients are still exposed to the risk of syringe swaps, which can account for up to $40 \%$ of medication errors. ${ }^{8}$

Recently, we published a randomized control trial evaluating an early prototype of the AVOID-error system. The study found that the AVOID-error system was able to produce complete compliance with "safe drug administration procedure "and thereby potentially reduce risk of both ampoule and syringe swaps. ${ }^{5}$ In response to the publication, many readers and reviewers raised primarily two main concerns about the initial prototype of the AVOID-error system. Firstly, patient safety concerns which may result from device failure or time delay in administration of urgent medications and secondly, that "forcing" actions may result in anaesthesia providers finding "workarounds" to bypass the system. ${ }^{9}$ To address the first concern of time delays and device failure, in the current iteration of the AVOID-error system we added a provision of an "Emergency Drug Administration Port" (Fig. 1b), which is simply a drug port accessible to the anaesthesia provider that allows for a route to administer drugs in case of failure of the AVOID-error system or in situations where time delay of 5 to 10 seconds in drug administration could lead to a deleterious outcome. In order to disincentivize the anaesthesia provider from finding workarounds to bypass the AVOID-error system, we updated the system software to allow it to integrate with commercial AIMS using their application program interfaces. Thus enabling the anaesthesia provider to enter the administered drug into the AIMS directly through the AVOID-error system, thereby making the documentation of administered drugs a more user friendly experience. An additional consequence of this AIMS-AVOID-error system integration is the ability of the AVOID-error system to detect any bypass attempts (workarounds) initiated by the anaesthesia provider. The improvement in the end-user experience because of this feature is apparent from the fact that around 35\% of participants felt that the AVOID-error system would not slow or may even improve current drug administration workflow, which contrasts with the first prototype. Finally, in our study $85 \%$ of the participants either expressed a willingness to use the AVOID-error system in their place of work $(65 \%)$ or had no unwillingness around the adoption of the system (20\%) (Fig. 4).
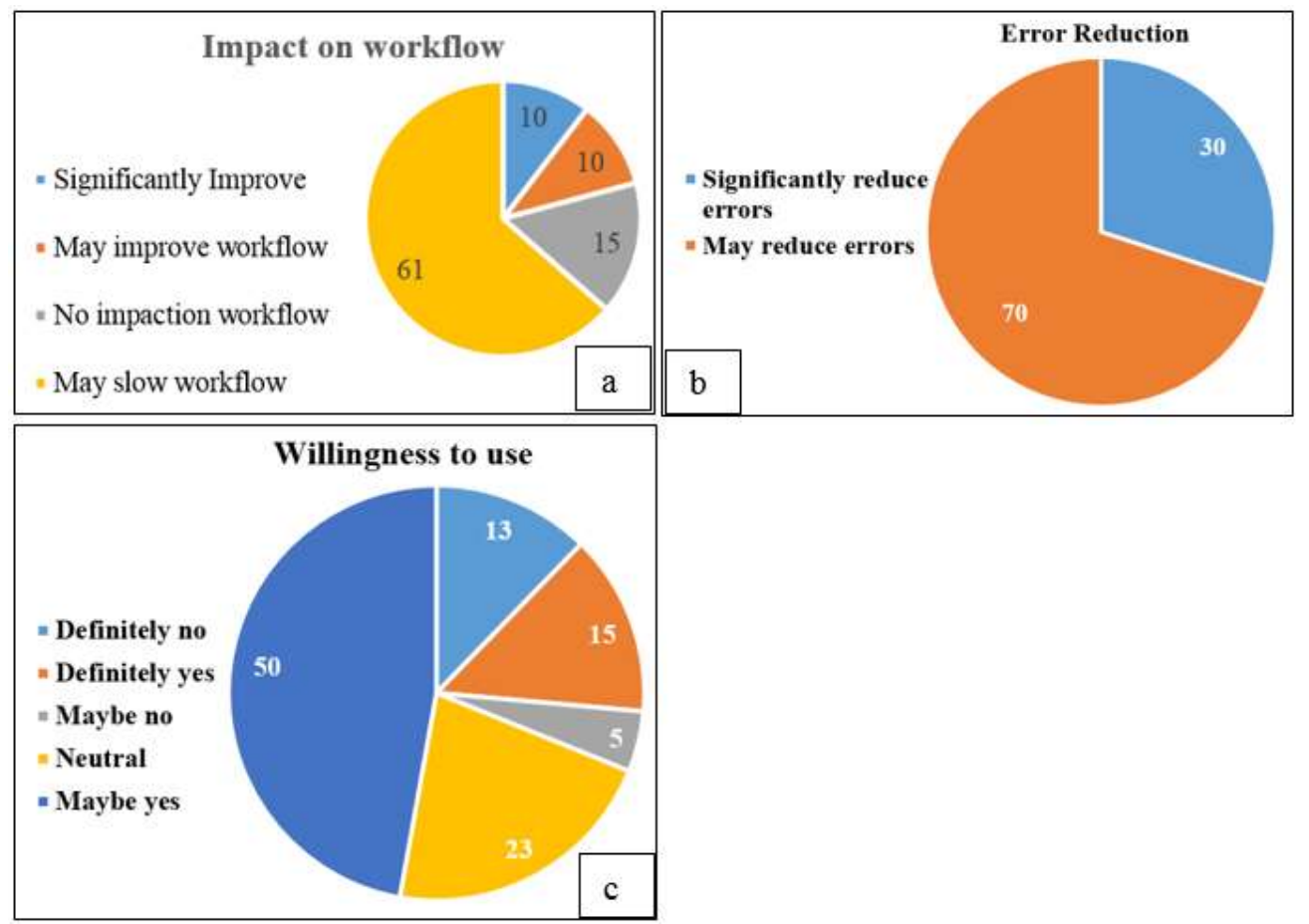

Fig. 4: Study participant's opinion regarding the impact of the AVOID-error system on: (4a): Workflow, (4b): Medication error reduction; (4c): Willingness to adopt in routine practice. All data presented as percentages 
Our study has some limitations. First, even though this study was a randomized controlled trial in an operating room like simulation environment, it was not a clinical study. The results of this study have however provided necessary information for us to plan a clinical evaluation of the AVOID-error system in the near future. Secondly, the study was not blinded because it was practically not possible, but in an effort to remove bias we did not reveal the primary study outcome to the participants. Lastly, based on our clinical opinion we chose a 5 second period to define significant time delay in drug administration. It can be argued that this period was too short or too long or should not have even been a issue taking into account the provision of the "Emergency Drug Administration Port" in the current iteration of the AVOIDerror system. But after taking the end-user and previous readers feedback into consideration, we felt that information about the time-delay in drug administration was essential for end-users to decide about potentially adopting the AVOIDerror system.

In conclusion, we found that even though the AVOIDerror system significantly increases the time to administer medication by 5.6 seconds (95\% CI, 4.6-6.5) compared to routine practice, there is considerable end-user opinion in favour of the AVOID-error system's ability to reduce the risk of medication errors and in willingness to adopt in routine practice.

\section{Conflict of Interest: None.}

\section{References}

1. Merry AF, Peck DJ. Anaesthetists, errors in drug administration and the law. N Z Med J 1995;108(1000):185-7.

2. Stelfox HT, Palmisani S, Scurlock C, Orav EJ, Bates DW. The "To Err is Human" report and the patient safety literature. Qual Saf Health Care 2006;15(3):174-8.
3. Nanji KC, Patel A, Shaikh S, Seger DL, Bates DW. Evaluation of Perioperative Medication Errors and Adverse Drug Events. Anesth 2016;124(1):25-34.

4. Weiser TG, Regenbogen SE, Thompson KD, Haynes AB, Lipsitz SR, Berry WR, et al. An estimation of the global volume of surgery: a modelling strategy based on available data. Lancet 2008;372(9633):139-44.

5. Khan SA, Khan S, Kothandan H. Simulator evaluation of a prototype device to reduce medication errors in anaesthesia. Anaesth 2016;71(10):1186-90.

6. Jelacic S, Bowdle A, Nair BG, Kusulos D, Bower L, Togashi K. A System for Anesthesia Drug Administration Using Barcode Technology: The Codonics Safe Label System and Smart Anesthesia Manager. Anesth Analg 2015;121(2):41021.

7. Merry AF, Webster CS, Hannam J, Mitchell SJ, Henderson R, Reid P, et al. Multimodal system designed to reduce errors in recording and administration of drugs in anaesthesia: prospective randomised clinical evaluation. $B M J$ 2011;343:d5543.

8. Fasting S, Gisvold SE. Adverse drug errors in anesthesia, and the impact of coloured syringe labels. Can J Anesth 2000;47(11):1060-7.

9. Webster CS, Merry AF. Forcing functions and their consequences. Anaesth 2017;72(2):266-7.

How to cite this article: Khan S, Chia P, Khan SA. Simulator evaluation of the additional verification of intravenous drug (AVOID) -error system: A prospective randomized controlled study. Indian J Clin Anaesth 2019;6(2):274-9. 